\title{
Die Synode von Serdika 343: Das Scheitern eines ökumenischen Konzils und seine Folgen für die Einheit der Reichskirche
}

\author{
Josef Rist
}

Am 28. Oktober des Jahres 312 wird am nördlichen Zugang zur Stadt Rom, in der weiten Ebene oberhalb des Tiber, in unmittelbarer Nähe zur Milvischen Brücke und kaum drei Kilometer von der Porta Flaminia entfernt, Weltgeschichte geschrieben. Der junge Konstantin, den christliche Historiographen bald schon den Großen nennen werden, wagt es, nach der Alleinherrschaft im Westen des Römischen Reiches zu greifen und seinen Widersacher Maxentius zur Entscheidungsschlacht herauszufordern. Das Wagnis gelingt: Maxentius verliert Schlacht und Leben, Konstantin triumphiert. ${ }^{1}$ Am Ende des denkwürdigen Tages ist Konstantin Herrscher über die Stadt Rom und den Westen des Imperiums. Im Jahr 324 kann er schließlich mit Licinius, dem Herrscher des Ostens, auch den letzten ernstzunehmenden Rivalen ausschalten und die Herrschaft über das Reich in seiner Hand vereinigen. Bereits nach der Schlacht an der Milvischen Brücke beginnt Konstantin das Christentum zu fördern. Es beginnt das, was als Konstantinisches Zeitalter zu einem viel diskutierten Epochenbegriff wird, die durch Konstantin eingeleitete enge Verbindung von Staat und Kirche. ${ }^{2}$

1 Eine materialreiche Darstellung der Schlacht und ihrer propagandistischen Instrumentalisierung bietet Wolfgang Kuhoff, "Ein Mythos in der römischen Geschichte: Der Sieg Konstantins des Großen über Maxentius vor den Toren Roms am 28. Oktober 312 n. Chr," Chiron 21 (1991), 27-174. Militärische Details: ebd. 147-162. Für die konstantinische Religionspolitik einschlägig sind die Arbeiten von Klaus Martin Girardet, insbesondere ders., Der Kaiser und sein Gott. Das Christentum im Denken und in der Religionspolitik Konstantins des Großen, Millennium-Studien 27 (Berlin, 2010).

2 Zu Ursprung, Konzeption und Kritik des Begriffs vgl. Christoph Markschies, "Wann endet das 'Konstantinische Zeitalter'?" in Die Weltlichkeit des Glaubens in der Alten Kirche. Festschrift Ulrich Wickert, ed. Dietmar Wyrwa, Beihefte zur Zeitschrift für die neutestamentliche Wissenschaft und die Kunde der älteren Kirche 85 (Berlin, 1997), pp. 157-188. Nach Wilhelm Schneemelder bezeichnet das Konstantinische Zeitalter die Jahre zwischen 312 (Schlacht an der Milvischen Brücke) und 380 (Edikt Cunctos populos des Kaisers Theodosius). Vgl. ebd. p. 169 .

(C) JOSEF RIST, 2015 | DOI 10.1163/9789004291935_006

This is an open access chapter distributed under the terms of the Creative Commons AttributionNoncommercial 3.o Unported (CC-BY-NC 3.o) License. 
Aus der Sicht nicht nur der Zeitgenossen schien nach diesen Ereignissen die Einheit des Reiches für absehbare Zeit sichergestellt. Die Herrschaft eines Kaisers, eine staatstragende Religion - aus christlicher Perspektive, etwa der des Kirchenhistorikers Eusebius von Caesarea, natürlich die christliche - und ein nach langen inneren Kämpfen endlich befriedetes Reich sollten sich ergänzen und gegenseitig stützen. ${ }^{3}$ Doch spätestens mit dem Tod des Kaisers im Jahr 337 holt die Realität diese Vorstellungen wieder ein. Ein erbitterter Kampf um die Macht entbrennt zwischen Konstantin II., Konstans und Konstantius II., den drei Söhnen des Kaisers, unter denen das Reichsgebiet vom Vater aufgeteilt wurde. Nicht zuletzt die von Konstantin eingeleitete enge Verbindung zwischen Staat und Kirche erweist sich jetzt als Bumerang und beschädigt angesichts der verbissenen Konflikte um die theologischen Positionen des alexandrinischen Presbyters Arius die erst vor kurzem gewonnene Einheit des Reiches.

Einen ersten Höhepunkt finden die theologischen Konflikte zwischen den beiden Reichsteilen in Serdika im Jahr 343. Ursprünglich als ökumenische Bischofsversammlung geplant, die die theologische Einheit des Reiches wiederherstellen sollte, wird hier der tiefe Bruch sichtbar, der sich nicht nur zwischen den an den Debatten beteiligten Kirchenparteien, sondern auch zwischen den beiden Reichsteilen aufgebaut hatte. Durch die Vorgänge in Serdika wird erstmals eine tiefe theologische Entfremdung innerhalb der Reichskirche zwischen Ost und West sichtbar. Im Laufe der Jahrhunderte setzt sich unter wechselnden politischen und theologischen Prämissen dieser Prozess fort und findet schließlich seinen Endpunkt in der demonstrativ erklärten Aufkündigung der Kirchengemeinschaft zwischen Rom und Byzanz im Jahr 1054. ${ }^{4}$

3 Zu Konstantins religionspolitischen Zukunftsplänen vgl. Girardet, Kaiser, pp. 150-163. Sein pointiertes, nicht unumstrittenes Resümee (ebd. p. 162): „Konstantins politische Intention ging also dahin, im Einklang mit dem Willen seines Gottes den religiösen Pluralismus im Reich und auf der Welt insgesamt allmählich zum Verschwinden zu bringen, das Christentum zur alleinigen Reichs- und Menschheitsreligion zu machen." Zu Eusebius vgl. Gerhard Ruhbach, "Die politische Theologie Eusebs von Caesarea," in Die Kirche angesichts der konstantinischen Wende, ed. ders., Wege der Forschung 306 (Darmstadt, 1976), pp. 236-58.

4 Vgl. Leslie W. Barnard, The council of Serdica 343 A.D. (Sofia, 1983), p. 5: "that the Council of Serdica ... marked the beginning of this process for there East and West separated by conciliar decision for the first time." Zum Schisma von 1054 und seiner Vorgeschichte vgl. Axel Beyer, Spaltung der Christenheit. Das sogenannte Morgenländische Schisma von 1054, Beihefte zum Archiv für Kulturgeschichte 53 (Köln, 2002). 


\section{Die Ausgangslage}

Spätestens ab dem Jahr 327 beginnt Kaiser Konstantin unter steter Beeinflussung seiner theologischen Entourage seine Haltung zum Arianismus zu verändern. Hatte er zuvor die gegen Arius und seine Lehre gerichteten Beschlüsse des ersten ökumenischen Konzils von Nizäa 325 bestätigt und die wenigen Bischöfe, die sich dieser Entscheidung entgegenstellen (unter ihnen Bischof Eusebius von Nikomedien), verbannt, so betreibt er nun aktiv die Versöhnung mit der arianischen Kirchenpartei. ${ }^{5}$ Bereits im Sommer 326 verlässt mit dem spanischen Bischof Ossius von Cordoba ein wichtiger theologischer Berater und Unterstützer des nizänischen Glaubens den Kaiserhof. Zielscheiben des nun einsetzenden Kampfes gegen die Gegner des Arius im Episkopat sind insbesondere die Bischöfe Eustathius von Antiochien (Datum der Absetzung umstritten:326 bzw.328, "Auftakt zu einerlängeren Reiheähnlicher Maßnahmen"6), Athanasius von Alexandrien ${ }^{7}$ und Markell von Ancyra. ${ }^{8}$ Über die Rechtgläubigkeit der beiden letztgenannten "Anti-'Eusebianer"'9 ${ }^{2}$ werden auch die in Serdika versammelten Bischöfe kontrovers diskutieren.

Wenige Bemerkungen mögen zur reichspolitischen Situation genügen. Mit dem Tod Konstantins am 22. Mai des Jahres 337 wird die Herrschaft im Römischen Reich wieder aufgeteilt. Die Konstantinssöhne Konstantin II., Konstantius II. und Konstans erhalten jeweils einen Teil des Imperiums als Herrschaftsgebiet zugesprochen. ${ }^{10}$ Schon bald kommt es zum Zerwürfnis unter den Brüdern. Als Ergebnis eines blutigen Bürgerkrieges kann der jüngste Sohn Konstans im Jahr 340 seinen älteren Bruder Konstantin II., der über den Nord-

Vgl. zu Kirchenpolitik Konstantins nach dem Konzil von Nikaia: Charles Pietri, Christoph Markschies, "Theologische Diskussionen zur Zeit Konstantins: Arius, der "arianische Streit" und das Konzil von Nizäa, die nachnizänischen Auseinandersetzungen bis 337," in Die Geschichte des Christentums. Religion-Politik-Kultur. Altertum 2: Das Entstehen der einen Christenheit (250-430), eds. Charles und Luce Pietri (Freiburg, 1999), pp. 317-340.

6 Ebd. 324 .

7 Standardwerk: Peter Gemeinhardt, ed., Athanasius Handbuch (Tübingen, 2011).

8 Vgl. Sara Parvis, Marcellus of Ancyra and the Lost Years of the Arian Controversy 325-345, Oxford Early Christian Studies (Oxford, 2006).

9 Pietri, Markschies, Theologische Diskussionen, p. 327. Namensgeber der Eusebianer ist wahrscheinlich Bischof Eusebius von Nikomedien.

10 Zu den Plänen Konstantins und ihrer Umsetzung vgl. Heinrich Chantraine, Die Nachfolgeordnung Constantins des Großen, Abhandlungen der Akademie der Wissenschaften und der Literatur, Mainz, Geistes- und Sozialwissenschaftliche Klasse, Jahrgang 1992, Heft 7 (Stuttgart, 1992) sowie Richard Klein, "Die Kämpfe um die Nachfolge nach dem Tode Constantins des Großen," Byzantinische Forschungen 6 (1979), 101-150. 
westen des Imperiums (Gallien, Britannien, Spanien) gebot, besiegen; dieser findet in den Kämpfen den Tod. Damit ist die von Konstantin projektierte Nachfolgeregelung für das Reich endgültig zerfallen. Jetzt stehen sich der Westen des Reiches unter der Regentschaft des Konstans und der Ostteil unter Konstantius II. abwartend gegenüber. ${ }^{11}$

Die Aufteilung des Reiches unter zwei Kaiser hat auch für den Episkopat gravierende Folgen; sie macht die Kirchenpolitik zunächst für alle Beteiligten "wesentlich komplizierter". ${ }^{2}$ Der theologische Konflikt um die Lehre des Arius und der kirchenpolitische Kampf um die Rechtgläubigkeit einzelner östlicher Bischöfe wie Athanasius und Markell von Ancyra verbindet sich eng mit dem machtpolitischen Ringen zwischen den beiden Reichsteilen um die Vorherrschaft. Dabei bemühen sich einerseits die verschiedenen Kirchenparteien um die Gunst der Kaiser, andererseits sind diese wiederum "massiv daran interessiert, mit Hilfe der Loyalität von Bischöfen einerseits die Machtposition im eigenen Herrschaftsgebiet zu stärken und andererseits im jeweils anderen Reichsteil an Einfluß zu gewinnen."13

In den Jahren vor den Synoden in Serdika steht die Person und Theologie des alexandrinischen Bischofs Athanasius im Mittelpunkt der kirchenpolitischen Auseinandersetzungen. Seit seiner umstrittenen Wahl zum Bischof von Alexandrien am 8. Juni 328 sind Konflikte die ständigen Begleiter des streitbaren Kirchenmannes. ${ }^{14}$ Neben lokalen ägyptischen Problemfeldern wird Athanasius insbesondere sein unerschütterliches Festhalten am nizänischen Glauben zum Vorwurf gemacht. Einen ersten Höhepunkt findet der Konflikt im Jahr 335, als Athanasius von einer in Tyrus versammelten Synode als Bischof abgesetzt und von Kaiser Konstantin in sein erstes Exil nach Trier geschickt wird. ${ }^{15}$ Der Streit um die kirchenrechtliche Gültigkeit des in Tyrus

11 Die häufig postulierte Vormundschaft Konstantin II. über seinen jüngeren Bruder darf heute als widerlegt gelten. $\mathrm{Zu}$ den komplexen Vorgängen des Jahres 340 vgl. Bruno Bleckmann, “Der Bürgerkrieg zwischen Constantin II. und Constans (340)," Historia 52 (2003), 225-250.

Charles Pietri, Jörg Ulrich, "Von der partitio des christlichen Kaiserreichs bis zur Einheit unter Konstantius: Arianerstreit und erster "Caesaropapismus"," in Die Geschichte des Christentums. Religion-Politik-Kultur. Altertum 2: Das Entstehen der einen Christenheit (250-430), eds. Charles und Luce Pietri (Freiburg, 1999), p. 348.

Vgl. Jörg Ulrich, Die Anfänge der abendländischen Rezeption des Nizänums, Patristische Texte und Studien 39 (Berlin, 1994), p. 29.

14 Kurze Biographie mit weiterführender Literatur: Tobias Georges, "Der Bischof von Alexandrien," in Athanasius Handbuch, ed. Peter Gemeinhardt (Tübingen, 2011), pp. 82-93.

15 Vgl. ebd. 85f. Ausführlicher zur Synode von Tyrus als vollkommener Ausdruck der konstantinischen Staat-Kirche-Beziehung: Klaus Martin Girardet, Kaisergericht und Bischofs- 
ergangenen Absetzungsurteils, von dessen Rechtmäßigkeit die östlichen Bischöfe überzeugt sind, bildet den Kern der in den folgenden Jahren mit großer Intensität einerseits von Athanasius, aber auch von seinen Gegnern geführten Auseinandersetzungen. Bleibt der Appell des alexandrinischen Kirchenmannes an Kaiser Konstantin zunächst erfolglos, so kann er jedoch bereits 337 nach dessen Tod gegen den ausdrücklichen Willen der östlichen Bischöfe ${ }^{16}$ mit Erlaubnis des Kaisers Konstantius II. nach Alexandrien zurück. Als schließlich der östliche Herrscher im Jahr 339 die Einsetzung des Kappadokiers Gregor als Bischof von Alexandrien billigt und Athanasius in einer recht rabiaten Polizeiaktion vertrieben wird, flieht Athanasius in den Westen und sucht Schutz und Unterstützung in Rom beim dortigen Bischof Julius. ${ }^{17}$

Der römische Bischof spielt für den weiteren Fortgang der komplexen Angelegenheit eine wichtige Rolle. In Rom wird Athanasius herzlich in die Gemeinschaft der Ortsgemeinde aufgenommen. Rasch entwickelt sich die Stadt zum Sammelpunkt zahlreicher östlicher Bischöfe, die sich durch die im Osten dominierenden Eusebianer ebenfalls in ihrer Treue zum Nizänum bedrängt und verfolgt sehen. Schließlich trifft auch Markell von Ancyra, in dessen Bischofsstadt es ebenfalls zu gewaltsamen Auseinandersetzungen gekommen ist, in Rom ein. ${ }^{18}$ Ziel des römischen Bischofs ist es, die Causa Athanasius erneut aufzurollen. Zu diesem Zweck lädt er eine östliche Delegation zu einer Synode nach Rom ein. ${ }^{19}$ Die Einladung ist mit einer Fristsetzung verbunden. Der Osten lehnt ab, was Julius aber nicht daran hindert, im Frühjahr 341 zusammen mit 50 anderen Bischöfen aus Italien die Bischofsversammlung abzuhalten. Ihr Ergebnis ist, dass sowohl Athanasius als auch Markell von Ankyra von den im

gericht. Studien zu den Anfängen des Donatistenstreites (313-315) und zum Prozeß des Athanasius von Alexandrien (328-346), Antiquitas 1/21 (Bonn, 1975), pp. 66-75.

Vgl. Sozomenos, Historia Ecclesiastica 3.2.8, eds. Joseph Bidez und Günther Christian Hansen (Berlin, 1995), p.103, line 14-16; Übersetzung: Günther Christian Hansen, Fontes Christiani 73,2 (Turnhout, 2004), p. 337: „Eusebius nämlich und seine Freunde versuchten ihn eifernd beim Kaiser (sc. Constantius) als Unruhestifter zu verleumden, der entgegen den

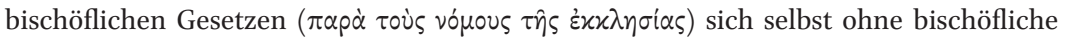
Bestätigung die Rückkehr angemaßt habe."

17 Vgl. Annick Martin, Athanase d'Alexandrie et l'Église d'Égypte au IVe siècle (328-373), Collection de l'École française de Rome 216 (Rom, 1996), pp. 403-409. Übersichtskarte zum Aufenthalt des Athanasius außerhalb Ägyptens: ebd. 397.

Vgl. Pietri, Ulrich, Von der partitio, p. 351. Eintreffen des Markell: "Ende 339 oder Anfang 340" (Ulrich, Anfänge, p. 32).

Der Brief des Julius ist nicht erhalten, wohl aber ein Regest bei Sozomenos, Historia Ecclesiastica 3.8.3, Athanasius Werke, eds. Hanns Christof Brennecke, Uta Heil, Annette von Stockhausen, Angelika Wintjes, 1. Teil, 3. Lieferung (Berlin, 2007), Nr. 41 (pp. 137f.). 
Osten gegen sie erhobenen Vorwürfen freigesprochen werden und die Kirchengemeinschaft mit ihnen bestätigt wird. Über dieses Vorgehen informiert der römische Bischof Julius die östlichen Amtsbrüder in einem ausführlichen Brief, den Athanasius überliefert, und der auf die östliche Verweigerung der Teilnahme an der Synode antwortet. ${ }^{20}$

$\mathrm{Zu}$ Recht hat Jörg Ulrich in seiner luziden Darstellung darauf hingewiesen, dass die beiden Streitfälle Athanasius und Markell von Ancyra höchst unterschiedlich gelagert sind. ${ }^{21}$ Beim Konflikt um Athanasius geht es primär um eine kirchenrechtliche Frage, nämlich die Gültigkeit der im Osten gegen den alexandrinischen Bischof erfolgten Synodalentscheidungen. Anders gelagert ist der Fall des Markell von Ankyra. Hier steht die umstrittene Theologie des Bischofs und damit seine Rechtgläubigkeit im Mittelpunkt der Angriffe. Noch zu Lebzeiten Konstantins war Markell, der eine höchst komplexe Trinitätslehre entwickelt und sich als Gegner der Eusebianer profiliert hatte, von einer Synode in Konstantinopel (Juli 336 oder Anfang 337) als Häretiker abgesetzt und exiliert worden. ${ }^{22}$ Dennoch bilden in der Wahrnehmung des Jahres 341 beide Streitfälle aus westlicher Sicht eine unauflösliche Einheit. Dies kommt im Brief des römischen Bischofs Julius deutlich zum Ausdruck: Das Schreiben entlastet den alexandrinischen Bischof von den diversen, auf den vorangegangen Synoden gegen ihn erhobenen, nicht dogmatischen Vorwürfen, während Markell vom Verdacht der Häresie freigesprochen wird. ${ }^{23}$ Damit hatte sich der

20 Der Brief des Julius bei Athanasius, Apologia (secunda) contra Arianos 21-35. Text und Übersetzung bei Brennecke, Athanasius Werke, Nr. 41.8 (pp. 156-175). Ausführliches Referat und Analyse: Girardet, Kaisergericht, pp. 87-105. Zur römischen Synode vgl. Martin, Athanase, pp. 410-419. Vgl. Ulrich, Anfänge, p. 27.

22 Die Nachrichten über diese Synode sind gesammelt bei Brennecke, Athanasius Werke, Nr. 40 (pp. 132-136). Zur Datierung und zum Verlauf vgl. Parvis, Marcellus, pp. 127-132. Bischofsliste: ebd. 259. Vgl. ebd. 131: "The deposition of Marcellus is irrefutable proof, if any more were needed, of the political brilliance of the Eusebian alliance." Zur konsequent trinitarisch gedachten Theologie des Markell vgl. Klaus Seibt, Die Theologie des Markell von Ankyra, Arbeiten zur Kirchengeschichte 59 (Berlin, 1994).

23 Vgl. die Zusammenfassung bei Ulrich, Anfänge, p. 33. Julius kann feststellen (Text: Brennecke, Athanasius Werke, p. 16o, line 11-22; Übersetzung: ebd.): „Denn die Häresie der Ariomaniten wurde von allen Bischöfen überall verurteilt und verboten, die Bischöfe Athanasius und Markell aber haben ziemlich viele, die für sie aussagen und schreiben. Denn über Markell wurde uns bezeugt, daß er auch auf der Synode in Nicaea denen, die arianisch dachten, widerstand, von Athanasius aber wurde uns bezeugt, dass er auch in Tyrus nicht verurteilt wurde, in der Mareotis aber nicht anwesend war, wo das belastende Material gegen ihn herstammt, wie es heißt. Ihr wißt aber, Geliebte, daß einseitiges Material keine Beweiskraft hat, sondern verdächtig erscheint.“ 
Westen eindeutig theologisch positioniert: Die Bischöfe um Julius erkennen die Theologie des aus dem Osten stammenden Markell als rechtgläubig an. Dies bedeutet nicht nur eine klare antiarianische Ausrichtung, sondern auch in Einzelfragen Kritik an Origenes, eine der zentralen theologischen Autoritäten des Ostens. ${ }^{24}$

In der Zwischenzeit hatte sich auch der Osten theologisch festgelegt. In Antiochien versammeln sich anlässlich einer Kirchweihe, die wohl 341 stattfindet, die östlichen Bischöfe. Auch Kaiser Konstantius II. ist persönlich auf der Synode anwesend. ${ }^{25}$ Mit der Synode stehen drei Glaubensbekenntnisse, die sogenannten drei antiochenischen Formeln, in direkter Verbindung. Auf der Grundlage der östlichen theologischen Tradition setzen sich die Glaubensformeln in sehr unterschiedlicher Intensität und Form mit dem Vorwurf des Arianismus auseinander, nähern sich dem Glauben von Nizäa an, betonen aber gleichzeitig die Ablehnung der als häretisch angesehenen Anschauungen des Markell. ${ }^{26}$ Damit sind die grundlegenden theologischen Ausgangspositionen benannt, die wenig später in Serdika aufeinander prallen sollten.

Dass es überhaupt zum Projekt einer gemeinsamen Synode aus Ost und West kommen sollte, verdankt sich neben dem verständlichen Wunsch der beiden Exilanten Athanasius und Markell nach Rehabilitation dem Eingreifen der beiden Kaiser. Treibende Kraft ist dabei der westliche Augustus Konstans. Er ist bereits früh über die Angelegenheit unterrichtet und wird wohl im Frühjahr 341 auch über die Ergebnisse der römischen Bischofsversammlung informiert. Ohne Zögern unterstützt er das Vorhaben einer Reichssynode und wendet sich in diesem Sinne an seinen Bruder. Eine östliche Delegation, die das Vorhaben durch eine persönliche Intervention am Hof des Konstans in Trier verhindern will, scheitert kläglich. Schließlich stimmt Konstantius zu. ${ }^{27}$ Zu Recht wird das Eigeninteresse des Konstantius am Zustandekommen der Synode hervorgehoben, sieht er doch hier eine Chance, über das Mittel der

24 Zur Haltung des Markell zu Origenes vgl. Seibt, Theologie, pp. 280-292.

25 Die sogenannte Kirchweihsynode ist nicht genau datierbar (zwischen dem 1. September 340 und dem 31. August 341). Es liegen keine Akten vor. Die Berichte sind gesammelt bei Brennecke, Athanasius Werke, Nr. 41.2 (pp. 138-142).

Vgl. Ulrich, Anfänge, pp. 37f. Texte: Brennecke, Athanasius Werke, Nr. 41.3 (pp. 143f.: dritte Formel), Nr. 41.4 (pp. 144-148: zweite Formel), Nr. 41.5 (pp. 148-150: erste Formel). Siehe auch Richard Patrick Crosland Hanson, The Search for the Christian Doctrine of God: The Arian Controversy 318-381 (Edinburgh, 1988), pp. 284-292. 110. 
Kirchenpolitik, d. h. durch die erneute Installation der in den Westen vertriebenen Bischöfe, Einfluss auf das östliche Reichsgebiet zu gewinnen. ${ }^{28}$

\section{Der Verlauf der Beratungen}

Als Datum der in Serdika stattfindenden Bischofsversammlung wird in der Literatur sowohl das Jahr 342 als auch - in jüngeren Publikationen verstärkt das Folgejahr 343 genannt. Zur Datierungsfrage wurde im Laufe der Jahrzehnte in der Forschung eine umfangreiche und kontroverse Debatte geführt. ${ }^{29}$ Die meisten vorgetragenen Argumente sind ebenso wie die ihnen zugrunde liegenden Texte mehrdeutig. Nach den neueren chronographischen Arbeiten von Richard Burgess neigt sich aber die Waage eindeutig zugunsten des Jahres 343. In ihrer ausgewogenen, alle Argumente erneut prüfenden Synthese kann Sarah Parvis zeigen, dass die Synode von Serdika aller Wahrscheinlichkeit nach im Jahr 343 stattgefunden hat. ${ }^{30}$

Über den Gang der Beratungen sind wir gut unterrichtet. Eine ganze Reihe einschlägiger Dokumente, die über die lokalen Ereignisse und ihre theologische Bewertung berichten, sind überliefert. Dazu zählen die Rundbriefe und theologischen Erklärungen der beiden Synoden sowie verschiedene Briefe. ${ }^{31}$ Zusätzlich hat Athanasius einige Zeit nach der Synode die Ereignisse aus seiner Perspektive niedergeschrieben. Erstaunlicherweise sind die Dokumente der östlichen Synode in Serdika, etwa die sogenannte theologische Erklärung der östlichen Synode, in lateinischer Sprache überliefert, während umgekehrt viele Schreiben der westlichen Synode eine wesentlich bessere griechische Überlieferung aufweisen. Dieser erstaunliche Befund wurde von Angelika Wintjes philologisch untersucht. Demnach erscheint es als wahrscheinlich, dass die Texte der östlichen Synode im Original griechisch abgefasst wurden. Ebenso lagen die Haupttexte der westlichen Synode zunächst in lateinischer Sprache vor, wurden aber wohl noch auf der Synode ins Griechische übersetzt. Auch sind mehrfache Übersetzungsprozesse einzelner Dokumente nicht aus-

28 So Ulrich, Anfänge, p. 39. Girardet spricht sogar von „einer politischen ... Erpressung“ (ders., Kaisergericht, p. 110) des Konstantius durch seinen Bruder.

29 Wichtige Beiträge sind für 342: Ulrich, Anfänge, pp. 39-44 sowie für 343: Barnard, Council, pp. $46-55$.

30 Vgl. mit ausführlicher Forschungsgeschichte und reichen Belegen: Parvis, Marcellus, pp. 210-217. Kurze Zusammenstellung der wichtigsten Argumente für 343 auch bei Brennecke, Athanasius Werke, pp. $179 f$.

Die Texte sind gesammelt, übersetzt und kommentiert bei Brennecke, Athanasius Werke, Nr. 43-43.13 (pp. 179-279). Ausführlich auch Barnard, Council, pp. 78-96. 
zuschließen. ${ }^{32}$ Einen Sonderfall bilden die Kanones von Serdika, die auf die westliche Synode zurückgehen und sich ausschließlich mit Fragen des Bischofsamtes beschäftigen. ${ }^{33}$

Das zukünftige Konzil soll drei Themenfelder behandeln, die von der westlichen Seite vorgegeben werden. ${ }^{34}$ Sie werden im Brief der westlichen Synode an den römischen Bischof Julius konkret benannt: zunächst die Glaubensfrage, dann, eng mit dieser verbunden, die Klärung der Rechtmäßigkeit der im Osten vorgenommenen Bischofsabsetzungen und schließlich weitere kirchenrechtliche Angelegenheiten. ${ }^{35}$

Die westliche Delegation trifft als Erste in Serdika ein. ${ }^{36}$ Konsequent der auf der römischen Synode beschlossenen Linie folgend, nehmen die Westler Athanasius und Markell sogleich in ihre Gemeinschaft auf und feiern mit ihnen Eucharistie. Die später eintreffenden östlichen Bischöfe müssen erkennen, dass die westliche Seite bereits vollendete Tatsachen geschaffen hat. Sie fordern als Voraussetzung für die Aufnahme der gemeinsamen Konzilsdebatten, dass die Westler zuvor die Verbannten aus ihrer Gemeinschaft und damit von den folgenden Verhandlungen ausschließen müssten, um so zunächst die östlichen Synodalbeschlüsse anzuerkennen. Als dies nicht geschieht, verweigern sie weitere Verhandlungen und ziehen sich in ihr Hauptquartier, den kaiserlichen Palast in Serdika, zu weiteren Beratungen zurück. "Die Synode von Serdika war damit in zwei Teilsynoden gespalten, ehe sie überhaupt begonnen hatte." 37

32 Vgl. Angelika Wintjes, "Die ursprachliche Fassung der Dokumente von Serdica, " in Von Arius zum Athanasianum. Studien zur Edition der «Athanasius Werke», ed. Annette von Stockhausen und Christof Brennecke, Texte und Untersuchungen zur Geschichte der altchristlichen Literatur 164 (Berlin-New York 2010), pp. 127 f.

$33 \mathrm{Zu}$ den Kanones und den mit ihnen verbundenen Problemen vgl. Heinz Ohme, "The Synod of Serdica (342)," in The History of Byzantine and Eastern Canon Law to 150o, eds. Wilfried Hartmann, Kenneth Pennington, History of medieval canon law 4 (Washington, D.C., 2012), pp. 66-74. Siehe auch Barnard, Council, pp. 97-118.

34 Vgl. Parvis, Marcellus, p. 229.

35 Vgl. Brief der westlichen Synode an Julius: Brennecke, Athanasius Werke, Nr. 43.5, 4 (pp. 225f.).

36 Die Ereignisse sind mehrfach dargestellt. Vgl. den Überblick bei Ulrich, Anfänge, pp. 44-47. Ausführlicher bei Hanns Christof Brennecke, Hilarius von Poitiers und die Bischofsopposition gegen Konstantius II. Untersuchungen zur dritten Phase des Arianischen Streites (337-361), Patristische Texte und Studien 26 (Berlin, 1984), pp. 29-46.

37 Ulrich, Anfänge, p. 45. Die westlichen Bischöfe nehmen in Serdika an verschiedenen Orten Unterkunft. Vgl. Parvis, Marcellus, p. 234. Ihre Tagungen finden wohl in einem Gebäude nahe der Georgs-Rotunde statt. Ausführlich dazu: L.W. Barnard, "The Council of Serdica - Two Questions Reconsidered," in Ancient Bulgaria. Papers Presented to the Inter- 
Wohl fehlt es von beiden Seiten nicht an Versuchen, einen Kompromiss zu finden, der die erneute Aufnahme der gemeinsamen Debatten ermöglicht hätte. So wird vorgeschlagen, dass eine paritätisch besetzte Bischofskommission nach Ägypten entsandt werden soll, um vor Ort die Richtigkeit der Anschuldigungen gegen Athanasius zu überprüfen. ${ }^{38}$ Dies wird aber von westlicher Seite strikt abgelehnt. Auch beklagen die Orientalen, dass die Stimmung in der Stadt Serdika ihnen gegenüber zunehmend feindseliger wird und die Gefahr von Unruhen bestehe. ${ }^{39}$ Doch auch von westlicher Seite scheint es Kompromissangebote gegeben zu haben. Zumindest berichtet der greise Bischof Ossius von Cordoba, einer der Anführer der westlichen Bischöfe in Serdika, davon in seinem Brief an Kaiser Konstantius, den Athanasius überliefert. ${ }^{40}$ Mehrfach habe er dessen Ankläger aufgefordert, ihre Anschuldigungen erneut vorzubringen, sei es vor dem versammelten Konzil oder aber vor ihm allein. Werde Athanasius für schuldig befunden, so solle er aus der kirchlichen Gemeinschaft ausgestoßen werden. Andernfalls ist Ossius bereit, ihn mit sich nach Spanien zu nehmen. Die östliche Seite lehnt ab. ${ }^{41}$ Damit ist der Plan einer Reichssynode, die die kirchliche Einheit wiederherstellen sollte, endgültig gescheitert.

Schließlich teilt Eustathius, ein lokaler Presbyter und enger Mitarbeiter des Ortsbischofs Protogenes, den westlichen Bischöfen den Beschluss ihrer östlichen Amtsbrüder mit, Serdika zu verlassen. Bevor die Bischöfe aber die Stadt verlassen und sich auf die Heimreise bewegen, haben sie noch vor Ort im kaiserlichen Palatium einige wichtige Entscheidungen getroffen, die berechtigen, auch von einem „Eastern council of Serdica“42 zu sprechen. Ein ausführlicher Synodalbrief rekapituliert den Konflikt um Athanasius und Markell und beschreibt die Vorgänge in Serdika bis hin zur Abreise der Orientalen. ${ }^{43}$ Im Paragraphen 28 des Schreibens werden die führenden Bischöfe der Gegenseite, allen voran der römische Bischof Julius („principem et ducem malorum ${ }^{44}$ ) exkommuniziert. Angehängt sind dem Schreiben die theologische Erklärung

national Symposium on the Ancient History and Archaeology of Bulgaria, University of Nottingham, 1981, ed. Andrew Poulter (Nottingham, 1983), pp. 215-220.

Vgl. den Rundbrief der östlichen Synode bei Brennecke, Athanasius Werke, Nr. 43.11,19 (p. 263).

39 Vgl. Parvis, Marcellus, p. 233.

40 Vgl. Athanasius, Historia Arianorum 44.2f.

41 Ulrich hält den Vorschlag des Ossius für ein Täuschungsmanöver. Vgl. Ulrich, Anfänge, p. 46 Anm. 146. Ähnlich skeptisch ist Barnard, Council, p. 67f.

42 Ebd. 68.

43 Text: Brennecke, Athanasius Werke, Nr. $43 \cdot 11$ (250-272).

44 Ebd. Nr. 43.11, 28 (270, 31). 
der östlichen Synode sowie eine Unterschriftenliste. ${ }^{45}$ Die Westler hielten sich nach der Abreise der Orientalen wohl noch eine Zeit in Serdika auf. Sie verfassen ebenfalls einen Synodalbrief sowie weitere Schriftstücke, bevor auch sie die Heimreise antreten. „Wohl noch vor dem Wintereinbruch ${ }^{46}$ wird die $\mathrm{Zu}$ sammenkunft beendet.

Ein kurzes Wort zum theologischen Inhalt der von den westlichen Bischöfen in Serdika beschlossenen Glaubenserklärung, dem sogenannten Serdicense: ${ }^{47}$ Das Symbolum betont die eine Hypostase des Vaters, des Sohnes und des Heiligen Geistes, um so Christus gegen arianische Verkürzungen als wahren Gott zu sichern. Abgelehnt und als Irrelehre verworfen wird deshalb die von Origenes begründete und von den Eusebianern vertretene Vorstellung von drei Hypostasen. ${ }^{48}$ Der mittlerweile von der Mehrheit der Forscher in seiner Authentizität nicht mehr angezweifelte Text besitzt zahlreiche Übereinstimmungen mit der theologischen Position des Markell von Ankyra, so dass Jörg Ulrich feststellen kann: „Das westliche Serdicense ist demnach dogmengeschichtlich als eine in allen wesentlichen Punkten mit der Lehre Markells übereinstimmende theologische Erklärung einzustufen. ${ }^{49}$

\section{Die Ursachen der Spaltung}

Um den Ursachen für die in Serdika gescheiterten Beratungen und das dadurch verursachte Schisma auf die Spur zu kommen, sind drei Fragenkomplexe zu untersuchen: 1.) Die Zusammensetzung der jeweiligen Bischofsgruppen. Auf den ersten Blick erscheint es so, als ob sich in Serdika geschlossene Blöcke aus den beiden Reichsteilen gegenüberstehen, es sich also um einen Konflikt handelt, der gleichzeitig eine klare geographische und theologische Trennlinie zwischen Ost und West abbildet. 2.) Die Rolle des römische Bischofs Julius. 3.) Das Verhalten der beiden Kaiser Konstans und Konstantius II. und seine Bedeutung für den Verlauf der Beratungen.

Zunächst ist die Zusammensetzung der in Serdika anwesenden Bischöfe genauer zu erheben. Durch die Subskriptionslisten sind wir gut über die

\footnotetext{
45 Text der theologischen Erklärung: Brennecke, Athanasius Werke, Nr. 43.12 (pp. 272-275). Die Unterschriftenliste: ebd. 43.13 (pp. 275-279).

46 Vgl. Ulrich, Anfänge, p. 46.

47 Text: Brennecke, Athanasius Werke, Nr. 43.2 (pp. 205-212). Ausführliche Analyse bei Ulrich, Anfänge, pp. $47-87$.

48 Vgl. ebd. 67 f.

49 Ebd. 9o. Ausführliche Analyse der Übereinstimmungen: ebd. 87-91. Zur Authentizität des Textes vgl. ebd. 98-106.
} 
Anwesenden informiert. Athanasius berichtet, dass die Gesamtzahl der Teilnehmer beider Synoden 170 betrug. Dabei dürften an der westlichen Teilsynode zwischen 90 und 97 Bischöfe, an der östlichen nach deren eigenen Angaben 80 Bischöfe, von denen 73 mit Namen bekannt sind, teilgenommen haben. ${ }^{50}$ Keiner der beiden Kaiser ist in Serdika anwesend. ${ }^{51}$ Rund ein Drittel der westlichen Bischöfe stammt aus Griechenland und den benachbarten Balkangebieten - Provinz Achaia 13, Macedonia 9, Creta 5 und Thessalia 4 -, während der eigentliche Westen (Spanien, Gallien, Italien, Afrika) nur sehr schwach vertreten ist. Die Verteilung der östlichen Bischöfe auf die jeweiligen Provinzen ist demgegenüber deutlich ausgeglichener. ${ }^{52}$ Aus den Namenslisten folgert Ulrich für die westliche Synode, dass "gut über 50 Teilnehmer der Synode und damit etwa 6o\% der Anwesenden von Hause aus griechisch sprachen," eine Beobachtung, die durch die Überlieferung der Synodaldokumente gestützt wird. ${ }^{53}$ Bedeutende Vertreter der östlichen Partei sind unter anderem die Bischöfe Stephan von Antiochien, Akakios von Caesarea und Menophantes von Ephesus, während aus der westlichen Gruppe der greise Ossius von Cordoba - geradezu die Inkarnation des nizänischen Glaubens -, der Ortsbischof Protogenes sowie Maximin von Trier besonders hervorstechen. ${ }^{54}$ Jedenfalls darf man sich die beiden Bischofsgruppen nicht als geschlossene Gruppen vorstellen. 55

Es bleibt festzuhalten, dass die sogenannte westliche Synode mehrheitlich von Bischöfen besetzt ist, die aus griechischsprachigen Gebieten des Imperiums stammen. Sie verhandeln eine dogmatische Frage, die als Folge der lange schwelenden arianischen Streitigkeiten ebenfalls ihren Ursprung im Osten hat und deren Hauptprotagonisten östliche Theologen sind. Besonders hervorzuheben ist, dass die von der westlichen Gruppe in Serdika im Symbolum, dem Serdicense, vertretene trinitätstheologische Position jene des Markell von Ankyra ist, also ebenfalls eines Theologen aus der östlichen Reichshälfte. Aus diesem Grund sind die zuweilen anzutreffenden Postulate eines sogenannten westlichen Bekenntnisses in Serdika nur als Unterscheidungsbegriffe zu der

50 Vgl. Athanasius, Historia Arianorum 15.3. Vgl. zu den Teilnehmern: Ulrich, Anfänge, pp. 91-96. Ausführliche Listen, auch aufgeschlüsselt nach Provinzen bei Brennecke, Athanasius Werke, pp. 180-184, Nützlich ist die beigegebene Karte, die die Bischofssitze der Teilnehmer verzeichnet: ebd. 185 .

Vgl. ebd. 180.

$5^{2} \quad$ Vgl. ebd. 184.

53 Ulrich, Anfänge, p. 95. Allerdings ist mit Wintjes (dies., Fassung, pp. 107-116) von einem lateinischen Prätext des Serdicense auszugehen.

54 Vgl. Barnard, Council, pp. 56-62.

55 Vgl. für die östliche Seite: Parvis, Marcellus, p. $232 \mathrm{f}$. 
östlich-origenistisch geprägten lokalen Teilsynode sinnvoll. Ähnliches gilt auch für den in diesem Beitrag mehrfach verwendeten Begriff westliche Synode. ${ }^{56}$

Eine Schlüsselrolle bei der Bewertung der Vorgänge in Serdika kommt dem römischen Bischof Julius (Amtszeit 337-352) zu. ${ }^{57}$ Ausgehend von den zeitgenössischen Bewertungen der östlichen Partei erscheint er in der Literatur als besonders belastet. Der römische Bischof selbst ist in Serdika nicht anwesend, die römische Position vertreten dort die beiden Presbyter Archidamus und Philoxenus sowie der Diakon Leo. Mit der athanasianischen Frage ist Julius bereits seit den Jahren 338 befasst, als Gesandtschaften sowohl aus Antiochien und später aus Alexandrien in Rom eintreffen, um, gestützt auf einschlägige Dokumente, darzulegen, weshalb Athanasius aus der kirchlichen Gemeinschaft auszuschließen sei oder nicht. Die Gegner des Athanasius scheitern. In diesem Zusammenhang taucht auch erstmals der Gedanke einer Synode zur Lösung der Streitigkeiten auf. ${ }^{58}$ Verwickelt wird Julius in den Konflikt durch die Initiative des Athanasius und des Markell, die die Unterstützung des römischen Bischofs für ihre Sache suchen. Mit der Einberufung der römischen Synode 341 tritt eine entscheidende Wende ein: Neben die theologischen Konflikte tritt nun die Frage, ob Julius berechtigt ist, sich zum Richter über östliche Synodalentscheidungen zu machen und die Konfliktparteien vor seine römische Synode zu zitieren. ${ }^{59}$ Hier tritt bei Julius offen ein primatiales Bewusstsein zu Tage. Der römische Bischof sieht wohl, ähnlich seinem Vorgänger in der kurz zuvor maßgeblich durch Rom entschiedenen Donatistenfrage, erneut die Chance, Ansehen und Bedeutung seines Stuhles zu steigern. Es gilt aber bei aller Kritik die Ausgangslage zu beachten: „der römische Bischof greift nicht zuerst aus eigener Initiative in die entstandenen Probleme ein. Vielmehr wendet man sich aus Ost und West an den römischen Stuhl, weil man von dort

$5^{6}$ Vgl. Ulrich, Anfänge, p. 96. Ulrich erinnert an die Warnung von Martin Tetz (zitiert ebd. 26 Anm. 1): „Man weiß, daß sich nicht einfach Osten und Westen, nicht Morgen- und Abendländer oder Griechen und Lateiner, auch nicht Origenisten und Antiorigenisten schieden."

Zur Person vgl. Manlio Simonetti, "Giulio I, santo," in Enciclopedia dei papi. Volume 1 (Rome, 2000), pp. 334-340. Siehe auch Wilhelm Gessel, "Das primatiale Bewusstsein Julius' I. im Lichte der Interaktionen zwischen der Cathedra Petri und den zeitgenössischen Synoden," in Konzil und Papst. Historische Beiträge zur Frage der Höchsten Gewalt in der Kirche. Festgabe für Herman Tüchle, ed. Georg Schwaiger (München, 1975), pp. $63^{-74}$.

$5^{8}$ Vgl. Girardet, Kaisergericht, p. 8of. Julius wird von den Parteien nicht als Richter angerufen; von einer möglichen Synode in Rom ist keine Rede.

59 Vgl. ebd. 84-87. 
eine kompetente Lösung der bestehenden Schwierigkeiten erwartete. ... Entscheidend ist, dass man durch den Rekurs nach Rom grundsätzlich eine höhere Autorität anrief und somit diese im Prinzip anerkannte."60 Julius wehrt sich gegen die Vorwürfe, indem er seine Fürsorge für die Einheit der Kirche und den rechten Glauben betont und auf den besonderen Rang der römischen Gemeinde verweist. ${ }^{61}$ Der Osten erkennt im Vorgehen des Julius aber eine unkanonische Neuerung und einen schwerwiegenden Eingriff in die grundsätzliche Gleichrangigkeit der Bischöfe. An den komplexen theologischen Debatten beteiligt sich Julius nicht. Durch die westliche Synode in Serdika erfolgt auch in kirchenrechtlicher Hinsicht eine Rangerhöhung des römischen Bischofs. Insbesondere durch Kanon 3, der Rom als Appellationsinstanz gegen die Entscheidung von Provinzialsynoden zulässt, werden die vorausgegangenen Aktionen Roms faktisch sanktioniert. ${ }^{62}$

Eine zentrale Rolle für die Vorgänge in Serdika spielt die politische Lage des unter zwei Kaisern aufgeteilten Reiches. Seit der Mission des comes Gabianus, der eine westliche Bischofsdelegation nach Antiochien im Jahr 341 begleitet, wo sie von den dort versammelten Amtskollegen die Wiedereinsetzung des Athanasius fordert, ist der westliche Kaiser Konstans aktiv in die Auseinandersetzungen eingebunden. Sein Ziel ist es, „den Rückhalt seines Bruders bei der Kirche zu schwächen.“63 Das politisch durchdachte, selbstsichere Auftreten und Agieren des Athanasius ist ohne die kaiserliche Rückendeckung nicht vorstellbar. Der Konflikt um Athanasius verwandelt die Machtfrage, d. h. das Ringen der beiden Herrscher um die Dominanz im Gesamtreich, in eine Glaubensfrage. Konstantius II. wird dabei zu Unrecht zum Parteigänger der Arianer gestempelt, seine theologischen Überzeugungen bewusst von westlicher Seite

\footnotetext{
$60 \quad$ Gessel, Bewusstsein, p. 69 .

61 Vgl. das (Verteidigungs-)Schreiben des Julius an die Synode von Antiochien. Text: Brennecke, Athanasius Werke, Nr. 41.8 (pp. 156-174). Dazu pointiert: Girardet, Kaisergericht, pp. 86f., 88-95.

62 Nach Girardet ist die Neuerung des Julius nicht die Überordnung seiner Synode über eine andere, sondern die Möglichkeit einer supplicatio, die sich nicht an eine Bischofssynode, sondern an einen einzigen Bischof, jenen von Rom, richtet. Vgl. Klaus Maria Girardet, "Appellatio. Ein Kapitel kirchlicher Rechtsgeschichte in den Kanones des vierten Jahrhunderts," Historia 23 (1974), 98-127. Zustimmend Brennecke, Hilarius, p. 44f. Zur Echtheitsfrage der Kanones vgl. Hamilton Hess, The Early Development of Canon Law and the Council of Serdica, Oxford Early Christian Studies (Oxford, 2002).

63 Richard Klein, Constantius II. und die christliche Kirche, Impulse der Forschung 26 (Darmstadt, 1977), p. 45 .
} 
verzeichnet. ${ }^{64}$ In dieser Perspektive erscheint Serdika als die Versammlung von zwei konkurrierenden Parteisynoden, deren eine die politische Unterstützung des östlichen Kaisers Konstantius II., die andere jene seines westlichen Pendants Konstans besitzt. ${ }^{65}$

Allerdings bildet sich die administrative Gliederung des Reiches in Ost und West nicht immer vollständig im Verhalten der Bischöfe in Serdika ab. So nimmt Bassus, der Bischof des thrakischen Diocletianopolis, an den Beratungen der westlichen Synode teil, während die aus derselben Provinz stammenden Amtskollegen Euticius von Philippopolis und Demophilus von Beroea zur östlichen Gruppe gehören. Wohl bestimmten kirchenpolitische Motive diese Wahl, konnte Bassus doch so den Eigenstand seines alten und einstmals bedeutenden Bischofssitzes gegenüber den Kollegen herausstellen. ${ }^{66}$ Auch treten persönliche Animositäten zuweilen hinter die theologische Generallinie zurück. Bischof Dionysius von Elis, Vorsteher einer Stadt in Achaia, ist Teil der westlichen Bischofsgruppe. Zu dieser gehören aber auch jene Amtskollegen, die ihn zuvor aus der kirchlichen Gemeinschaft ausgeschlossen hatten. ${ }^{67}$ Auf der anderen Seite stehen eigene theologische Anschauungen hinter dem Primat der Politik zurück. Ein Beispiel für diese Haltung scheint Bischof Protogenes von Serdika zu sein, der neben Ossius von Cordoba eine der wichtigsten Persönlichkeiten der westlichen Synode ist. ${ }^{68} \mathrm{Zu}$ Zeiten der Alleinherrschaft des Kaisers Konstantin ist er im Jahr 336 Teilnehmer einer Synode in Konstantinopel, die die Theologie des Markell von Ancyra verurteilt. Nach der Reichsteilung 337 befindet sich sein Bischofssitz aber im Reichsteil des Konstans, was die Tendenz zur Unterstützung der westlichen Position sicherlich verstärkt haben dürfte. ${ }^{69}$ Der Einfluss der Politik ist jedenfalls bei allen theologischen Ereignissen im Umfeld des Konzils von Serdika wahrzunehmen.

64 Vgl. ebd. pp. 46-51 zur Dominanz der Macht - über die Glaubensfrage bei Athanasius siehe ebd. p. $5^{1}$ Anm. 114 .

65 Vgl. Ulrich, Anfänge, p. 107.

66 So Barnard, Council, p. 58f. In den Unterschriftslisten wird Diocletianopolis wohl irrtümlich als Bischofssitz der Macedonia zugeordnet.

67 Vgl. Turhan Kaçar, "The Parting of the Ways: The East and the West at the Council of Serdica A.D. 343," Hacettepe Üniversitesi, Edebiyat Fakültesi Dergisi 19.1 (2002), 139-151, hier 144; Timothy David Barnes, Athanasius and Constantius. Theology and Politics in the Constantinian Empire (Cambridge, Mass., 1993), p. 74.

68 Vgl. Stanislava Stoycheva, "Bishop Protogenes of Serdica. His life and theological views (335-351)," Byzantinoslavica 60 (1999), 308-314.

69 So Kaçar, Parting, p. 145: „Only a political explanation may be offered for the attitude of Protegenes." 


\section{Schlussfolgerung}

Das Projekt eines in Serdika zu veranstaltenden Reichskonzils verdankt seine Grundlage verschiedenen Motiven. Im Mittelpunkt der theologischen Auseinandersetzungen steht die Frage nach der Rechtgläubigkeit des Athanasius von Alexandrien und des Markell von Ancyra. Die Bischöfe des östlichen Reichsteils lehnen in ihrer Mehrheit auf der Grundlage der origenistisch-eusebianischen Dreihypostasenlehre die Lehren des Markell ab und fordern die Anerkennung der in Tyrus 335 erfolgten Absetzung des Athanasius. Der durch den römischen Bischof Julius auf starkes Drängen des Athanasius beschrittene Weg einer Revision der östlichen Synodalbeschlüsse führt schließlich in Verbindung mit dem Wunsch des Kaiser Konstans, auch auf den Osten des Reiches auszugreifen, zum Plan eines ökumenischen Reichskonzils am Schnittpunkt der beiden Reichsteile in Serdika. Hier kommt es aus theologischer Perspektive nur vordergründig zur Konfrontation von Ost und West, stammt doch nur eine Minderheit der in Serdika anwesenden sogenannten westlichen Bischöfe tatsächlich aus dem lateinisch sprechenden Westteil des Reiches. Die theologische Debatte wird zudem von Athanasius und Markell, zwei Exilanten aus dem Osten, bestimmt.

Der Plan des Reichskonzils scheitert fast zwangsläufig. Jede der Bischofsgruppen, Ost und West, tagt separat und exkommuniziert die Gegenseite. Serdika ist kein Konzil, sondern wahrlich ein Debakel. ${ }^{70}$ Die Folgen für die Einheit der Reichskirche sind weitreichend: Wohl kommt es bereits kurze Zeit später zu Vermittlungsversuchen und die Alleinherrschaft des Konstantius ab dem Jahr 350 verändert erneut die politische Großwetterlage, dennoch hat der in Serdika angerichtete Schaden langfristige Folgen. Erstmals in der Kirchengeschichte haben sich, wenn auch durch Exilanten aus dem Osten verursacht, die Ortsgemeinden in Ost und West durch offizielle Beschlüsse voneinander getrennt, wobei die Differenzen „machtpolitischer (Konkurrenz der beiden Kaiser), kirchenpolitischer und kirchenrechtlicher (Frage der Berechtigung der Revision und ggf. Aufhebung von östlichen Synodalurteilen durch westliche Synoden) und dogmatischer Natur waren (Arianismus-Vorwurf gegen die Orientalen; Unannehmbarkeit der Theologie Markells aus Sicht der östlichen, der Tradition des Origenes verpflichteten Bischöfe) ${ }^{“}{ }^{71}$ In Serdika konnten sich

70 Vgl. Hanson, Search, p. 295: „The Council of Serdica never met as a Council. It was in fact a débacle rather than a Council, and it is absurd to reckon it among the General Councils, whether we look at it from the point of view of the Western or that of the Eastern bishops."

$71 \quad$ Ulrich, Anfänge, p. $46 f$. 
Ost und West in einer zentralen Frage des Glaubens nicht einigen, die kirchliche Einheit des Reiches war ebenso brüchig geworden wie die politische, die Entfremdung zwischen den Reichsteilen wird auch im Episkopat der Reichskirche für alle sichtbar. In diesem Sinne sind die Ereignisse in Serdika eine bedeutsame Zäsur in der Kirchengeschichte des vierten Jahrhunderts. ${ }^{72}$

\section{Bibliography}

Leslie W. Barnard, The council of Serdica 343 A.D. (Sofia, 1983).

, "The Council of Serdica - Two Questions Reconsidered," in Ancient Bulgaria. Papers Presented to the International Symposium on the Ancient History and Archaeology of Bulgaria, University of Nottingham, 1981, ed. Andrew Poulter (Nottingham, 1983), pp. 215-220.

Timothy David Barnes, Athanasius and Constantius. Theology and Politics in the Constantinian Empire (Cambridge, Mass., 1993).

Axel Beyer, Spaltung der Christenheit. Das sogenannte Morgenländische Schisma von 1054, Beihefte zum Archiv für Kulturgeschichte 53 (Köln, 2002).

Bruno Bleckmann, "Der Bürgerkrieg zwischen Constantin II. und Constans (340)," Historia $5^{2}$ (2003), 225-250.

Hanns Christof Brennecke, Hilarius von Poitiers und die Bischofsopposition gegen Konstantius II. Untersuchungen zur dritten Phase des Arianischen Streites (337-361), Patristische Texte und Studien 26 (Berlin, 1984), pp. 29-46.

Heinrich Chantraine, Die Nachfolgeordnung Constantins des Großen, Abhandlungen der Akademie der Wissenschaften und der Literatur, Mainz, Geistes- und Sozialwissenschaftliche Klasse, Jahrgang 1992, Heft 7 (Stuttgart, 1992).

Richard Patrick Crosland Hanson, The Search for the Christian Doctrine of God:The Arian Controversy $318-381$ (Edinburgh, 1988).

Hamilton Hess, The Early Development of Canon Law and the Council of Serdica, Oxford Early Christian Studies (Oxford, 2002).

Peter Gemeinhardt, ed., Athanasius Handbuch (Tübingen, 2011).

Tobias Georges, "Der Bischof von Alexandrien," in Athanasius Handbuch, ed. Peter Gemeinhardt (Tübingen, 2011), pp. 82-93.

Wilhelm Gessel, "Das primatiale Bewusstsein Julius' I. im Lichte der Interaktionen zwischen der Cathedra Petri und den zeitgenössischen Synoden," in Konzil und Papst.

72 Vgl. das spätere Urteil des Kirchenhistorikers Sokrates (ders., Historia ecclesiastica 2.22.2:

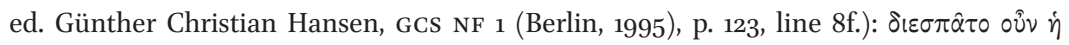

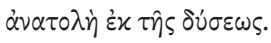


Historische Beiträge zur Frage der Höchsten Gewalt in der Kirche. Festgabe für Herman

Tüchle, ed. Georg Schwaiger (München, 1975), pp. 63-74.

Klaus Maria Girardet, Kaisergericht und Bischofsgericht. Studien zu den Anfängen des

Donatistenstreites (313-315) und zum Prozeß des Athanasius von Alexandrien (328-346), Antiquitas 1/21 (Bonn, 1975), pp. 66-75.

- "Appellatio. Ein Kapitel kirchlicher Rechtsgeschichte in den Kanones des vierten Jahrhunderts," Historia 23 (1974), 98-127.

- ders., Der Kaiser und sein Gott. Das Christentum im Denken und in der Religionspolitik Konstantins des Großen, Millennium-Studien 27 (Berlin, 2010).

Turhan Kaçar, "The Parting of the Ways: The East and the West at the Council of Serdica

A.D. 343," Hacettepe Üniversitesi, Edebiyat Fakültesi Dergisi 19.1 (2002), 139-151.

Richard Klein, Constantius II. und die christliche Kirche, Impulse der Forschung 26 (Darmstadt, 1977).

— , "Die Kämpfe um die Nachfolge nach dem Tode Constantins des Großen," Byzantinische Forschungen 6 (1979), 101-150.

Wolfgang Kuhoff, "Ein Mythos in der römischen Geschichte: Der Sieg Konstantins des Großen über Maxentius vor den Toren Roms am 28. Oktober $312 \mathrm{n}$. Chr," Chiron 21 (1991), 27-174.

Christoph Markschies, "Wann endet das 'Konstantinische Zeitalter'?," in Die Weltlichkeit des Glaubens in der Alten Kirche. Festschrift Ulrich Wickert, ed. Dietmar Wyrwa, Beihefte zur Zeitschrift für die neutestamentliche Wissenschaft und die Kunde der älteren Kirche 85 (Berlin, 1997), pp. 157-188.

Annick Martin, Athanase d'Alexandrie et l'Église d'Égypte au IV siècle (328-373), Collection de l'École française de Rome 216 (Rom, 1996), pp. 403-419

Heinz Ohme, "The Synod of Serdica (342)," in The History of Byzantine and Eastern Canon Law to 150o, eds. Wilfried Hartmann, Kenneth Pennington, History of medieval canon law 4 (Washington, D.C., 2012), pp. 66-74.

Sarah Parvis, Marcellus of Ancyra and the Lost Years of the Arian Controversy 325-345, Oxford Early Christian Studies (New York, 2006).

Charles Pietri and Christoph Markschies, "Theologische Diskussionen zur Zeit Konstantins: Arius, der "arianische Streit" und das Konzil von Nizäa, die nachnizänischen Auseinandersetzungen bis 337," in Die Geschichte des Christentums. ReligionPolitik-Kultur. Altertum 2: Das Entstehen der einen Christenheit (250-430), eds. Charles und Luce Pietri (Freiburg, 1999), pp. 317-340.

Charles Pietri and Jörg Ulrich, "Von der partitio des christlichen Kaiserreichs bis zur Einheit unter Konstantius: Arianerstreit und erster 'Caesaropapismus," in Die Geschichte des Christentums. Religion-Politik-Kultur. Altertum 2: Das Entstehen der einen Christenheit (250-430), eds. Charles und Luce Pietri (Freiburg, 1999), pp. 345-395. 
Gerhard Ruhbach, "Die politische Theologie Eusebs von Caesarea," in Die Kirche angesichts der konstantinischen Wende, ed. ders., Wege der Forschung 306 (Darmstadt, 1976), pp. 236-58.

Klaus Seibt, Die Theologie des Markell von Ankyra, Arbeiten zur Kirchengeschichte 59 (Berlin, 1994).

Manlio Simonetti, "Giulio I, santo," in Enciclopedia dei papi. Volume 1 (Rome, 2000), pp. 334-340.

Stanislava Stoycheva, "Bishop Protogenes of Serdica. His life and theological views (335-351)," Byzantinoslavica 60 (1999), 308-314.

Jörg Ulrich, Die Anfänge der abendländischen Rezeption des Nizänums, Patristische Texte und Studien 39 (Berlin, 1994).

Angelika Wintjes, "Die ursprachliche Fassung der Dokumente von Serdica," in Von Arius zum Athanasianum. Studien zur Edition der $<<$ Athanasius Werke $>>$, ed. Annette von Stockhausen und Christof Brennecke, Texte und Untersuchungen zur Geschichte der altchristlichen Literatur 164 (Berlin-New York 2010), pp. 105-129. 